\title{
Diagnosis and Treatment of Acute Gout at a University Hospital Emergency Department
}

\author{
Naomi Schlesinger ${ }^{*},{ }^{\text {, Diane C. Radvanski }}{ }^{1}$, Tina C. Young ${ }^{2}$, Jonathan V. McCoy ${ }^{3}$, \\ Robert Eisenstein ${ }^{3}$ and Dirk F. Moore ${ }^{2}$ \\ ${ }^{I}$ Division of Rheumatology, Department of Medicine, Rutgers Robert Wood Johnson Medical School, New Brunswick, \\ $N J, U S A$ \\ ${ }^{2}$ Department of Biostatistics, Rutgers School of Public Health Piscataway, NJ, USA \\ ${ }^{3}$ Department of Emergency Medicine, Rutgers Robert Wood Johnson Medical School, New Brunswick, NJ, USA
}

\begin{abstract}
Background: Acute gout attacks account for a substantial number of visits to the emergency department (ED). Our aim was to evaluate acute gout diagnosis and treatment at a University Hospital ED.

Methods: Our study was a retrospective chart review of consecutive patients with a diagnosis of acute gout seen in the ED 1/01/2004 - 12/31/2010. We documented: demographics, clinical characteristics, medications given, diagnostic tests, consultations and whether patients were hospitalized. Descriptive and summary statistics were performed on all variables.

Results: We found 541 unique ED visit records of patients whose discharge diagnosis was acute gout over a 7 year period. $0.13 \%$ of ED visits were due to acute gout. The mean patient age was 54; 79\% were men. For $118(22 \%)$ this was their first attack. Attack duration was $\leq 3$ days in $75 \%$. Lower extremity joints were most commonly affected. Arthrocentesis was performed in $42(8 \%)$ of acute gout ED visits.

During $355(66 \%)$ of ED visits, medications were given in the ED and/or prescribed. An anti-inflammatory drug was given during the ED visit during 239 (44\%) visits. Medications given during the ED visit included: NSAIDs: 198 (56\%): opiates 190 (54\%); colchicine 32 (9\%) and prednisone $32(9 \%)$. During 154 (28\%) visits an anti-inflammatory drug was prescribed. Thirty two $(6 \%)$ were given no medications during the ED visit nor did they receive a prescription. Acute gout rarely $(5 \%)$ led to hospitalizations.

Conclusion: The diagnosis of acute gout in the ED is commonly clinical and not crystal proven. Anti-inflammatory drugs are the mainstay of treatment in acute gout; yet, during more than $50 \%$ of ED visits, anti-inflammatory drugs were not given during the visit. Thus, improvement in the diagnosis and treatment of acute gout in the ED may be required.
\end{abstract}

Keywords: Acute gout, diagnosis, emergency department, treatment.

\section{INTRODUCTION}

Gout is the most common inflammatory arthritis in humans; affecting 8.3 million Americans [1]. Gout results from deposition of monosodium urate (MSU) crystals in and around the joints due to hyperuricemia [2,3]. There are several clinical stages resulting from hyperuricemia. These include: asymptomatic hyperuricemia, acute gout, intercritical gout (intervals between acute attacks) and chronic tophaceous gout due to MSU crystal deposition.

Acute gout is characterized by rapid onset and build-up of pain associated with warmth, redness and swelling of the affected joints. Wortmann [4] used the analogy "Gout is like matches" likening an acute attack to "setting the joint on fire". Lower extremity joints are most commonly involved. If untreated, attacks can last up to several weeks $[2,3]$.

*Address correspondence to this author at the Department of Medicine, Rutgers- Robert Wood Johnson Medical School, New Brunswick, NJ, USA; Tel: 1-732-235-8378; Fax: 1-732 235-7238;

E-mail: schlesna@rwjms.rutgers.edu
The gold standard for establishing a definite diagnosis of gout has been demonstrating the presence of MSU crystals in aspirated joint fluid or tophus [2,3]. However, physicians do not routinely perform synovial fluid analysis, even in hospitalized patients with acute gout [5] opting instead to reach a diagnosis based on clinical features and demonstration of hyperuricemia. Supportive clinical data to make a diagnosis of gout include a typical clinical history of a sudden and severe exquisitely painful joint most classically, the first metatarsophalangeal (MTP) joint and/or lower extremity joint/s; or a history of underlying renal disease or being on medications that can cause hyperuricemia and a favourable response to antiinflammatory drugs and topical ice [6].

Reports of poor and inappropriate diagnosis and treatment of acute gout attacks in different settings has been previously published $[5,7]$. This includes medication errors and initiation of a ULT during an acute gout attack [5].

The incidence of gout is increasing and represents a major health burden [8]. It is, therefore, not surprising that 
gout attacks account for a substantial number of ED visits [9]. Using the National ED Sample (NEDS) data Garg et al. [10] reported gout related healthcare utilization in United States EDs from 2006 through 2008. Acute gout was the primary reason for $168,410 \mathrm{ED}$ visits in $2006,171,743$ visits in 2007 , and 174,823 visits in $2008(0.2 \%$ of all ED visits annually) [10].

Our aim was to evaluate diagnosis and treatment of acute gout at a University Hospital ED.

\section{METHODS}

\section{Patient Selection}

Our study was an IRB-approved retrospective chart review of consecutive patient visits diagnosed at discharge from a university-based ED with acute gout who were seen between $1 / 01 / 2004$ and 12/31/2010. Included in the study were all patients seen in the ED, by emergency medicine faculty during the study period with a discharge diagnosis of acute gout.

We used methodological strategies to enhance validity, reproducibility and overall quality of data collected from the ED medical records in accordance with Gilbert's recommendations [11]. Emergency room electronic charts were printed out. The abstractors were the leading author (NS) and a Research assistant (DR). Charts were assigned a de-identified number. Variables were precisely defined; standardized abstraction forms were used for data collection; periodic meetings were held to review questions regarding data abstraction. A subsample of charts abstracted by the senior author was compared with the abstraction of the Research assistant.

\section{Variable Definition}

Variables included were: gender, age, race, systolic and diastolic blood pressures, history of hypertension, diabetes mellitus, history of gout, history of heart disease, medications given during the ED visit and those given as a prescription, consultations requested, physician assistant involvement, patient reported running out of medications, number of joints involved, attack duration, presence of tophi, diagnostic tests including: joint aspirations, x-rays, blood work, consultations and hospitalizations.

Descriptive and summary statistics were performed on all variables. Continuous variables were compared using linear regression and categorical variable were assessed using chi square tests. Sensitivity analysis and exploratory data analysis were performed where applicable.

\section{RESULTS}

We found 541 unique ED visits of patients whose discharge diagnosis was acute gout over a 7 year period. The ED volume during the study period was: 418,937 patients. Thus $0.13 \%$ of ED visits were due to acute gout. The mean patient age was 54 (range: 20-96); 425 (79\%) were males. Recorded comorbidities included: hypertension in 225
(43\%), diabetes mellitus in $96(18 \%)$ and heart disease in 85 $(16 \%)$. For $118(22 \%)$ patient visits this was their first gout attack while $391(77.3 \%)$ had a prior attack. Of those with prior attacks, $130(24 \%)$ had $\geq 1$ attacks in the past year. In $75 \%$ of ED visits duration of attack was $\leq 3$ days $(4.30 \pm$ SD 6.83 days). The most commonly affected joints were those of the lower extremities: first metatarsophalangeal (MTP) $(n=243)$, feet $(n=54)$ and knees $(n=108)$. The mean number of involved joints was $1.4( \pm \mathrm{SD} 1.046)$.

Arthrocentesis of a knee was performed in $42(8 \%)$ of acute gout ED visits. MSU crystals were found in $29(5 \%)$. Plain x-rays were done during $196(36 \%)$ of acute gout ED visits. The radiological reading was: normal in $161(82 \%)$; osteoarthritis (OA) in $27(14 \%)$; fracture in $2(1 \%)$ and not recorded in $6(3 \%)$. Seventy two $(13 \%)$ had blood drawn during the ED visit. Probability of having a blood draw increased with age $(p<.0001)$. Serum urate (SU) levels were checked in $72(13 \%)$.

Consultations were requested during $55(10 \%)$ of ED visits: $22(40 \%)$ orthopedic, $12(21.8 \%)$ rheumatologic, 17 $(30.9 \%)$ other services. Consultations were significantly more likely in older patients, when more joints were involved $(\mathrm{p}=0.003)$, and when there was $\geq 1$ comorbidity $(\mathrm{p}=0.03)$.

Medications were given during the ED visit in 355 (66\%) visits (Fig. 1). Medications included: NSAIDs: 198 (56\%)

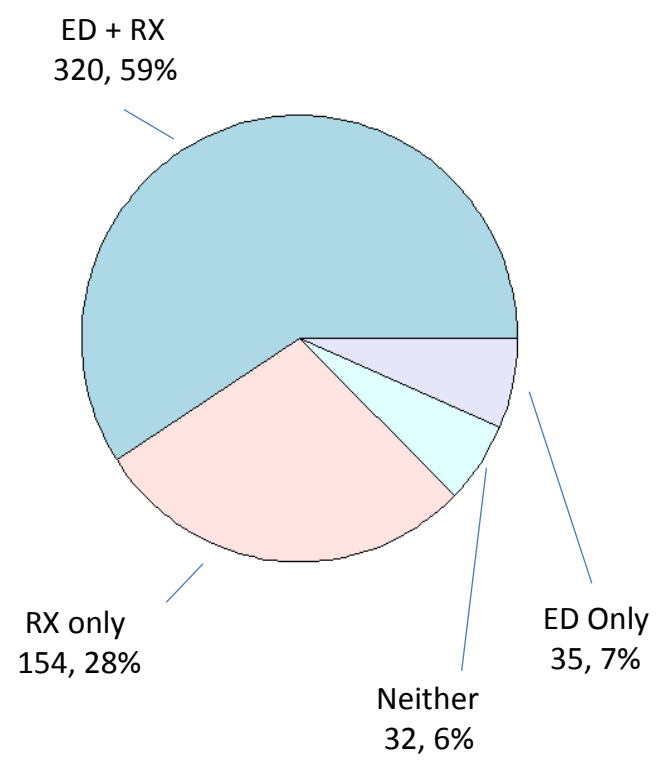

Fig. (1). Numbers and percentages of patients receiving treatment in the ED. Patients receiving medication and a prescription are denoted by "ED + RX"; patients receiving a prescription only are denoted by "RX only"; patients receiving medication only are denoted by "ED only"; and patients receiving neither medication nor a prescription are denoted by "Neither".

ketorolac 69 (19\%); indomethacin 66 (19\%); ibuprofen 49 (14\%) and naproxen 11 (3\%); opiates 190 (54\%); colchicine $32(9 \%)$ and prednisone $32(9 \%)$. Combination therapy was given during $92(17 \%)$ of visits. Combinations included: an 
NSAID plus oxycodone/acetaminophen in $36(10 \%)$; an NSAID plus colchicine in $13(4 \%)$ and prednisone plus oxycodone/acetaminophen in $11(3 \%)$. No corticosteroid injections were documented to have been given in the ED. One hundred fifty-four (28\%) not given medications during their ED visit were given a prescription. Thirty two $(6 \%)$ were given no medications during the ED visit nor did they receive a prescription. During $72(13 \%)$ of visits, the patient reported being on colchicine prophylaxis. Patients on colchicine prophylaxis were significantly more likely to receive treatment with colchicine for their acute attack than those not receiving prophylaxis $(\mathrm{p}=0.005)$.

As shown in Table 1, patients were given antiinflammatory drugs (AID) either in the ED, as a prescription, or both, or neither. An anti-inflammatory drug was given during 239 (44\%) of visits (Fig. 2) and 408 (75\%) given an anti-inflammatory drug prescription. Two hundred and sixteen $(40 \%)$ were given an anti-inflammatory drug in the ED and a prescription. $110(20 \%)$ were not given an antiinflammatory drug in the ED nor did they receive a prescription. Patients receiving anti-inflammatory drugs were younger $(\mathrm{p}=0.0008)$ and had a shorter attack duration prior to being seen in the ED ( $\mathrm{p}=0.0329)$. One hundred and ninety (35\%) were given an opiate (oxycodone/ acetaminophen) during the ED visit and $282(52 \%)$ a prescription for opiates. A change in urate lowering drugs (ULDs) (allopurinol and febuxostat) prescribing occurred in 7 (1\%) of $541 \mathrm{ED}$ visits. ULDs were started during 2 visits and stopped during 5 visits.

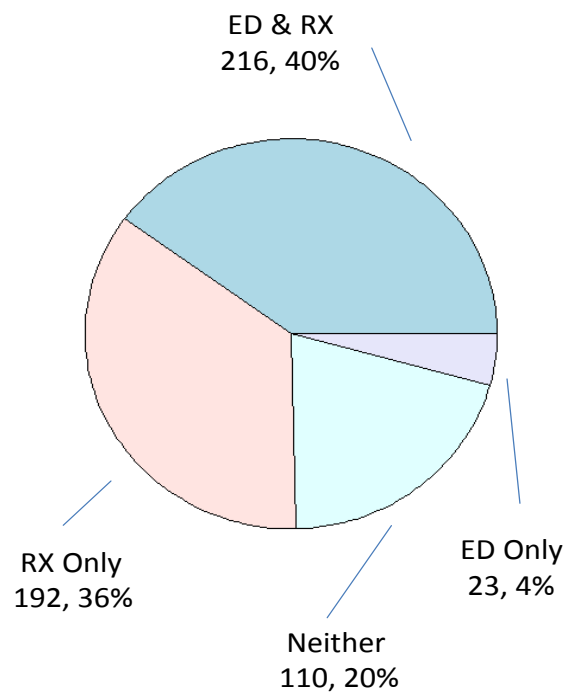

Fig. (2). Numbers and percentages of patients given antiinflammatory drugs (AID) or prescriptions for an acute gout attack. Patients receiving both AID and a prescription are denoted by "ED \& RX"; patients receiving a prescription only are denoted by "RX"; patients receiving AID only are denoted by "ED only"; and patients receiving neither are denoted by "Neither."

Seventy five (14\%) had their GFR (glomerular filtration rate) measured during the ED visit. Of 362 (66.7\%) patients given NSAIDs, 33 (9.1\%) had their GFR measured (4: GFR $<30 \mathrm{~mL} / \mathrm{min} / 1.73 \mathrm{~m}^{2}$ 5: GFR $30-59 \mathrm{~mL} / \mathrm{min} / 1.73 \mathrm{~m}^{2}$ in 4 patient visits). Those who received NSAIDs were less likely to have their GFR measured compared to those who didn't receive NSAIDs $(\mathrm{p}<.0001)$. Of $85(15.7 \%)$ patients given colchicine, 5 (6\%) had GFR checked (1: GFR 30-59 $\mathrm{mL} / \mathrm{min} / 1.73 \mathrm{~m}^{2} ; 4$ : GFR $\geq 60 \mathrm{~mL} / \mathrm{min} / 1.73 \mathrm{~m}^{2}$ ). Those who received colchicine were less likely to have GFR measured compared to those who did not receive colchicine $(\mathrm{p}=0.02)$. Twenty five $(5 \%)$ of acute gout ED visits ended in a hospitalization. In most hospitalizations $(19 ; 76 \%)$, the admitting diagnosis was acute gout.

\section{DISCUSSION}

We report the diagnosis and treatment of acute gout in a large series of 541 consecutive ED visits over a 7 year period. In this study, the diagnosis of gout was clinical in most patients. Arthrocentesis was rarely performed and when it was performed, it was done in knees of patients some suspected to have septic joint. In our study MSU crystals were rarely observed (5\%). The rarity of making a crystal diagnosis in patients suspected of having acute gout is consistent with other reports in the literature [12]. In Choi et al.'s study published in the New England Journal of Medicine only 11 percent of patients underwent arthrocentesis and only 65 percent of those were reported as having MSU crystals in the synovial fluid. Thus, only 7 percent of patients in their study had evidence of MSU crystals to confirm gouty arthritis, similar to the number found in our study. Clinical diagnosis is not optimal. Obtaining a definite diagnosis of gout via the gold standard of finding MSU crystals in the synovial fluid or tophaceous material is preferred $[13,14]$. This is most important in patients for whom this is the first gout attack. However, it is important to note that, no studies have compared cost and outcome of crystal-proven diagnosis versus clinical diagnosis in determining the outcome of care.

In the ED, plain $\mathrm{x}$-rays of joints, were commonly performed (36\%), however, they have a low sensitivity for gout and are rarely beneficial in its diagnosis. Most x-rays $(85 \%)$ were read as normal and none had findings suggestive of gout. Nevertheless, the x-rays may have been performed in some patients due to concerns for an alternate diagnosis such as osteomyelitis especially in older patients and in patients with multiple comorbidities, since we found the ordering rates to be significantly higher in those patients.

Consultations were requested in a minority $(10 \%)$ of acute gout patients presenting to the ED and rarely from the rheumatology service. A previous study showed that rheumatology consultations in the inpatient setting improved the treatment of acute gout [15]. As expected, consultations were significantly more frequent in sicker, older patients with more joints involved $(\mathrm{p}=0.003)$, and among patients with more than one comorbidity $(\mathrm{p}=0.036)$.

Checking the serum urate (SU) level during an acute attack should be reserved until after the resolution of a gout attack, as was the case in most patients seen in the ED for acute gout, since SU levels are often within the normal range during an acute attack [16] due to acute uricosuria that accompanies Interleukin (IL)-1 and IL-6 mediated gouty inflammation. In this study, SU levels were measured during $13 \%$ of acute gout ED visits, similarly to the rate observed in Garg's study (16\%) [10]. This study retrospectively reviewed electronic records of ED patients who visited the $\mathrm{ED}$, therefore, we do not have data on previous or recent 
Table 1. Demographics and Patient Comorbidities.

\begin{tabular}{|c|c|c|c|c|c|c|}
\hline Variable & Total & $\begin{array}{l}\text { Given AID in ED } \\
\text { and as Rx (Both) }\end{array}$ & $\begin{array}{l}\text { Given AID in ED } \\
\text { Only or as Rx Only }\end{array}$ & $\begin{array}{l}\text { Given non-AID in } \\
\text { ED and/or as Rx }\end{array}$ & $\begin{array}{l}\text { Given } \\
\text { No Drug }\end{array}$ & p-Value \\
\hline Total & 541 & $216(39.9 \%)$ & $215(39.7 \%)$ & $78(14.4 \%)$ & $32(5.9 \%)$ & \\
\hline \multicolumn{7}{|l|}{ Gender } \\
\hline $\mathrm{N}$ & 541 & 216 & 215 & 78 & 32 & \multirow{3}{*}{0.3454} \\
\hline Male & $425(78.6 \%)$ & $173(80.1 \%)$ & $167(77.7 \%)$ & $63(80.8 \%)$ & $22(68.7 \%)$ & \\
\hline Female & $116(21.4 \%)$ & $43(19.9 \%)$ & $48(22.3 \%)$ & $15(19.2 \%)$ & $10(31.3 \%)$ & \\
\hline \multicolumn{7}{|l|}{ Age } \\
\hline $\mathrm{N}$ & 541 & 216 & 192 & 78 & 32 & \multirow{3}{*}{$<.0001$} \\
\hline Mean (SD) & $54.43(15.545)$ & $51.68(14.999)$ & $55.24(15.428)$ & $58.23(15.214)$ & $58.31(17.938)$ & \\
\hline Min; Median; Max & $20 ; 54 ; 96$ & $21 ; 50 ; 96$ & $22 ; 55 ; 93$ & $26 ; 61 ; 85$ & $20 ; 55 ; 85$ & \\
\hline \multicolumn{7}{|c|}{ Presence of Diabetes Mellitus } \\
\hline $\mathrm{N}$ & 540 & 215 & 215 & 78 & 32 & \multirow{3}{*}{0.5212} \\
\hline Yes & $96(17.8 \%)$ & $34(15.8 \%)$ & $42(19.5 \%)$ & $14(18 . \%)$ & $6(18.8 \%)$ & \\
\hline No & $444(82.2 \%)$ & $181(84.2 \%)$ & $173(80.5 \%)$ & $64(82 . \%)$ & $26(81.3 \%)$ & \\
\hline \multicolumn{7}{|c|}{ Presence of Hypertension } \\
\hline $\mathrm{N}$ & 529 & 211 & 209 & 78 & 31 & \multirow{3}{*}{0.0017} \\
\hline Yes & $225(42.5 \%)$ & $74(35.1 \%)$ & $93(44.5 \%)$ & $41(52.6 \%)$ & $17(54.8 \%)$ & \\
\hline No & $304(57.5 \%)$ & $137(64.9 \%)$ & $116(55.5 \%)$ & $37(47.4 \%)$ & $14(45.2 \%)$ & \\
\hline \multicolumn{7}{|c|}{ Presence of Heart Disease } \\
\hline $\mathrm{N}$ & 524 & 209 & 207 & 78 & 30 & \multirow{3}{*}{0.1528} \\
\hline Yes & $85(16.2 \%)$ & $27(12.9 \%)$ & $37(17.9 \%)$ & $16(20.5 \%)$ & $5(16.7 \%)$ & \\
\hline No & $439(83.8 \%)$ & $182(87.1 \%)$ & $170(82.1 \%)$ & $62(79.5 \%)$ & $25(83.3 \%)$ & \\
\hline \multicolumn{7}{|c|}{ Presence of Any Comorbidity } \\
\hline $\mathrm{N}$ & 540 & 215 & 215 & 78 & 32 & \multirow{3}{*}{0.0228} \\
\hline Yes & $267(49.4 \%)$ & $93(43.3 \%)$ & $111(51.6 \%)$ & $46(59 \%)$ & $17(53.1 \%)$ & \\
\hline No & $273(50.6 \%)$ & $122(56.7 \%)$ & $104(48.4 \%)$ & $32(41 \%)$ & $15(46.9 \%)$ & \\
\hline \multicolumn{7}{|c|}{ Patient Reported Diet Change } \\
\hline $\mathrm{N}$ & 541 & 216 & 215 & 78 & 32 & \multirow{3}{*}{0.2135} \\
\hline Yes & $22(4.1 \%)$ & $10(4.6 \%)$ & $10(4.7 \%)$ & $2(2.6 \%)$ & $0(0 \%)$ & \\
\hline No & $519(95.9 \%)$ & $206(95.4 \%)$ & $205(95.3 \%)$ & $76(97.4 \%)$ & $32(100 \%)$ & \\
\hline
\end{tabular}

Ethnicity not included since reported in less than $10 \%$.

arthrocentesis, SU levels and GFR or creatinine levels. The issue of SU level measurements, especially when hyperuricemia is found, underscores the importance of viewing gout as a chronic disease and not just treating the acute attack. We would never release a patient who came in with a severe hyperoglycemic episode without attempting to ensure further follow-up for their diabetes mellitus; nor should we do so in the acute gout patient.

Health services research has shown that the number of ED visits due to disease exacerbations may be indicative of sub-optimal chronic disease management and poor treatment adherence $[17,18]$. In our study, sub-optimal chronic gout treatment including under-use and sub-optimal dosing of ULDs as well as infrequent use of chronic colchicine (13\%) and/or NSAIDs (19\%) for prophylaxis are suspected to have contributed to recurrent ED visits.

The goal of treatment of acute gout is prompt and safe termination of pain and inflammation. Anti-inflammatory drugs are the mainstay of treatment for an acute attack [19]. The most important determinant of therapeutic success is how soon treatment was started and that adequate dosing and duration of treatment was given. First-line anti-inflammatory drugs for the treatment of acute gout include oral colchicine, NSAIDs and corticosteroids [20], yet during over half (56\%) of acute gout ED visits, anti-inflammatory drugs were not given. It may be that some of the patients tried taking NSAIDs without relief prior to their ED visit. Furthermore, $20 \%$ of acute gout visits ended without being given an anti- 
inflammatory drug in neither the ED or as a prescription while $6 \%$ were not given medications at all. It is possible, that patients were given recommendations for medications that were not documented in the charts and that in some patients the attack had already peaked and was declining. Colchicine was infrequently utilized in the ED (given in only $9 \%$ of acute gout visits). The potential toxicity of colchicine may have led to it rarely being used for acute gout in the ED even prior to the US colchicine controversy [21]. In addition, the high prevalence of comorbidities in patients with gout may have limited the use of NSAIDs and colchicine in the ED.

Combination of colchicine and NSAIDs was suggested for severe or unresponsive attacks by the recent ACR guidelines [20]. Combination anti-inflammatory treatment was previously reported as being used by $50 \%-64 \%$ of rheumatologists $[5,22]$. In our study, combination treatment was used during $17 \%$ of ED patient visits. In most (76\%) of ED visits where combination treatment was given an opiate was used in combination with an NSAID or prednisone. However, there is little evidence to support combination treatment with opiates when treating an acute gout attack.

Our study has several strengths. Most importantly, it reflects current practice and is a large ED-based cohort with over $500 \mathrm{ED}$ acute gout visits and a long study duration of 7 years. There are several limitations to this study including its retrospective design, single-center site and the reliance on a clinical diagnosis of gout by the ED physicians (the diagnosis was rarely crystal proven), which may have led to some missed cases of acute gout or other diagnoses established on follow-up visits. While gout features identified by the Delphi Exercise by Prowse et al. [23], are a first step in establishing new gout classification criteria and, may help identify which patients better fit key gout features; ours is a retrospective study completed prior to Prower's publication, lacking some of the needed documentation. In addition, due to the retrospective nature of the study design, the study may have lacked documentation regarding of what drugs patients had tried prior to arriving at the ED and whether patients were instructed to use over the counter antiinflammatory medications when discharged from the ED.

The ED is a hectic, environment where patients present with high-acuity illness. ED physicians may be pressured to decrease ED length of stay [24] and may de-prioritize treatment of acute gout due to concerns about treating patients with more life-threatening conditions, although many times less ill patients are managed via a different work flow than more acute patients. This might explain the judicious use of opiates in acute gout. Opiates comprised approximately half of medications given during the ED visit and as prescriptions; however, use of analgesia alone does not treat underlying gout inflammation. Furthermore, there may be a perception that many patients presenting to the ED for pain control would not be satisfied with solely a prescription for medication that is already available to them over the counter, such as NSAIDs. Studies are needed to assess the use of opiates in the treatment of acute gout.

In conclusion, the diagnosis of acute gout in the ED is commonly clinical and not crystal proven. Antiinflammatory drugs are the mainstay of treatment in acute gout; yet, during more than $50 \%$ of ED visits, anti- inflammatory drugs were not given during the visit. Thus, improvement in the diagnosis and treatment of acute gout in the ED may be required.

\section{CONFLICT OF INTEREST}

Naomi Schlesinger, MD: Grants: Novartis; Advisory boards: Novartis, Takeda, Sobi; Speakers bureau: Novartis, Takeda; Consultant: Novartis, Sobi, BMS.

Diane C. Radvanski, MS: Grant Novartis.

Tina C. Young, MS, DrPH(c): Currently an employee of BMS (employment with Bristol-Myers Squibb began after completion of the work in this manuscript).

Jonathan V. McCoy, MD: None.

Robert Eisenstein, MD: Grant Novartis.

Dirk F. Moore, PhD: No disclosures.

\section{ACKNOWLEDGEMENTS}

Funding for this investigator initiated research provided by Novartis Pharmaceuticals Corporation. The funding organization had no role in the design and conduct of the study; data collection; analysis and interpretation of the data; or preparation or approval of the manuscript.

\section{REFERENCES}

[1] Zhu Y, Pandya B, Choi H. Prevalence of hyperuricemia in the US general population: the National Health and Nutrition Examination Survey (NHANES) 1999-2008. Arthritis Rheum 2011; 63(10): 3136-41.

[2] Schlesinger N. Diagnosis of gout. Minerva Med 2007; 98(6): 75967.

[3] Schlesinger N. Diagnosis of gout: clinical, laboratory, and radiologic findings. Am J Manag Care 2005; 11(Suppl 15): 443-50; quiz 465-8.

[4] Wortmann RL. Effective management of gout: an analogy. Am J Med 1998; 105: 513-4.

[5] Petersel D, Schlesinger N. Treatment of acute gout in hospitalized patients. J Rheumatol 2007; 34: 1566-8.

[6] Schlesinger N. Response to application of ice may help differentiate between gouty arthritis and other inflammatory arthritidies. J Clin Rheumatol 2006; 12: 275-6.

[7] Neogi T, Hunter DJ, Chaisson CE, et al. Frequency and predictors of inappropriate management of recurrent gout attacks in a longitudinal study. J Rheumatol 2006; 33: 104-9.

[8] Wallace KL, Riedel AA, Joseph-Ridge $\mathrm{N}$, et al. Increasing prevalence of gout and hyperuricemia over 10 years among older adults in a managed care population. J Rheumatol 2004; 31(8): $1582-7$.

[9] Singh JA, Sarkin A, Shieh M, et al. Health care utilization in patients with gout. Semin Arthritis Rheum 2011; 40: 501-11.

[10] Garg R, Sayles HR, Yu F, et al. Gout-related healthcare utilization in u.s. emergency departments, 2006 through 2008. Arthritis Care Res 2012; 65(4): 571-7.

[11] Gilbert EH, Lowenstein SR, Koziol-McLain J, et al. Chart reviews in emergency medicine research: where are the methods? Ann Emerg Med 1996; 27(3): 305-8.

[12] Choi HK, Atkinson K, Karlson EW, Willett WC, Curhan G. Purine-rich foods, dairy and protein intake, and the risk of gout in men. N Engl J Med 2004; 350: 1093-103.

[13] Malik A, Schumacher HR, Dinnella JE, Clayburne GM. Clinical diagnostic criteria for gout: comparison with the gold standard of synovial fluid crystal analysis. J Clin Rheumatol 2009; 15(1): 22-4.

[14] Janssens HJEM, Janssen M, van de Lisdonk EH, van Riel PLCM, van Weel C. Limited validity of the American College of Rheumatology criteria for classifying patients with gout in primary care. Ann Rheum Dis 2010; 69: 1097-102. 
[15] Barber C, Thompson K, Hanly JG. Impact of a rheumatology consultation service on the diagnostic accuracy and management of gout in hospitalized patients. J Rheumatol 2009; 36(8): 1699-704.

[16] Schlesinger N, Watson DJ, Norquist JM. Serum urate during acute gout. J Rheumatol 2009; 36(6): 1287-9.

[17] Sarawate CA, Brewer KK, Yang W, et al. Gout medication treatment patterns and adherence to standards of care from a managed care perspective. Mayo Clin Proc 2006; 81(7): 925-34.

[18] Singh JA, Hodges JS, Toscano JP, et al. Quality of care for gout in the US needs improvement. Arthritis Rheum 2007; 57(5): 822-9.

[19] Schlesinger N. Management of acute and chronic gouty arthritis: present state-of-the-art. Drugs 2004; 64(21): 2399-416.

[20] Khanna D, Khanna PP, FitzGerald JD, et al. American college of rheumatology guidelines for management of gout part II: therapy and anti-inflammatory prophylaxis of acute gouty arthritis. Arthritis Care Res 2012; 64(10): 1447-61

[21] Woodcock J, Okada S. Incentives for drug development - the curious case of colchicine. N Engl J Med 2010; 363: 1484-5.

[22] Schlesinger N, Moore DF, Sun JD, et al. A survey of current evaluation and treatment of gout. J Rheumatol 2006; 33: 2050-2.

[23] Prowse RL, Dalbeth N, Kavanaugh A, et al. A Delphi exercise to identify characteristic features of gout — opinions from patients and physicians, the first stage in developing new classification criteria. J Rheumatol 2013; 40; 498-505.

[24] Morris ZS, Boyle A, Beniuk K, et al. Emergency department crowding: towards an agenda for evidence-based intervention. Emerg Med J 2012; 29(6): 460-6.

(C) Schlesinger et al.; Licensee Bentham Open.

This is an open access article licensed under the terms of the Creative Commons Attribution Non-Commercial License (http://creativecommons.org/licenses/by-nc/ 3.0/) which permits unrestricted, non-commercial use, distribution and reproduction in any medium, provided the work is properly cited. 